\title{
A Humming Lattice of Cold Atoms
}

\author{
Researchers have produced an optical lattice of atoms that can generate \\ sound, a previously unachieved feat.
}

By Katherine Wright

$\Gamma$

he movements and interactions of ultracold atoms, trapped by lasers in a periodic lattice structure, provides a popular analog for the behaviors of electrons in crystalline solids (see Coming Soon: Cold Atoms Impersonate Superconductors). But the ultracold atomic system lacks a key feature of solid materials-the lattice on which the atoms sit can't vibrate or deform. Studies that rely on the analogous optical lattice fail to capture the impact on the system of natural vibrations intrinsic to condensed-matter systems. Now, Benjamin Lev of Stanford University and colleagues demonstrate a way to add that motion to the lattice. Lev says that the advance could enable researchers to better replicate and explore the behavior of condensed-matter systems using cold atoms, as well as to create novel forms of quantum matter.

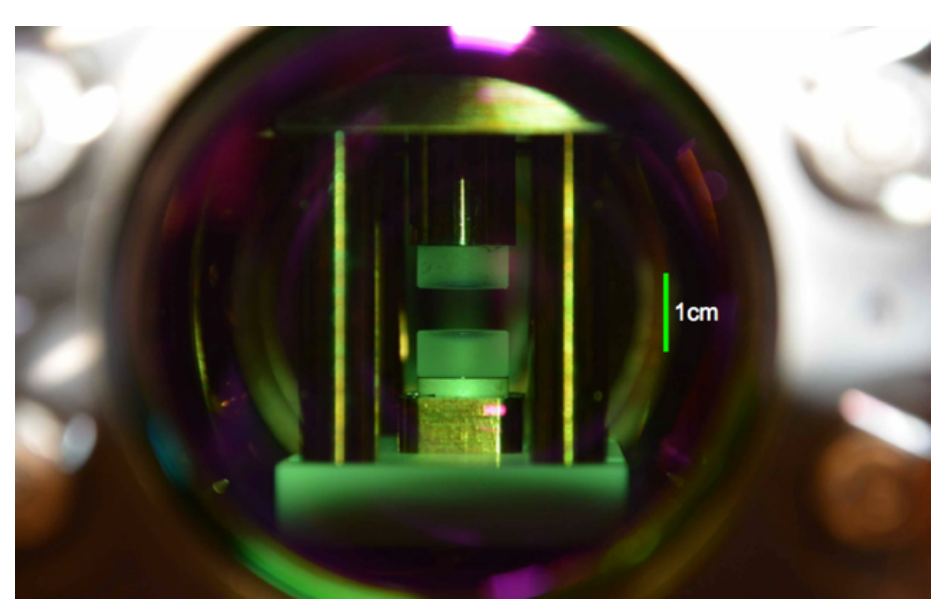

A tool for adding elasticity to an optical lattice could enable researchers to create novel states of matter.

Credit: B. Lev/Stanford University
Optical lattices are static, rigid structures by design.

Traditionally made by interfering laser beams in free space, they can also be produced in optical cavities, which confine standing light waves. The result is a checkerboard light field comprising high- and low-intensity regions. An atom in the cavity that wanders into a high-intensity "square" when this field is turned on experiences an attractive force that draws it to the center of that square and traps it in place. The optical cavities used in such experiments are known as single-mode cavities, meaning that only one specific wavelength can be confined as a standing wave. As a result, any lattice made in such a cavity has a fixed and rigid structure. "The spacing between the nodes [of the lattice] can't bend or vibrate," Lev says. In other words, the lattice is silent.

To generate sound, an optical lattice needs flexibility. Predictions suggest that creating the lattice in a multimode optical cavity may introduce that flexibility. Multimode cavities, as their name implies, support many different light patterns, all made with the same frequency of light. Creating lattices using different patterns adds compliance to the lattice. "The cavity we use provides a lot more flexibility in terms of the shape of the light that bounces back and forth between the mirrors," Lev says. "It's as if instead of just being allowed to make a single wave in a trough of water, you can now splash about to make any sort of wave pattern." That means that "if the atoms want to change their positions a little bit, the light can accommodate those changes without much difficulty," he adds.

Multimode cavities exist but have rarely been used in cold-atom experiments. Now, demonstrating this new application, Lev and his colleagues confined 100,000 ultracold rubidium atoms 
inside a multimode cavity that consists of two curved mirrors, each with radius of curvature equal to the length of the cavity.

The team's calculations show that photons that resonate in such a cavity can, when scattering off the trapped atoms, mediate interactions between those atoms through an exchange of photons. This exchange induces a flexing of the lattice. Theory shows that this flexing mimics the movement of atoms in solids caused by quantized sound waves known as phonons. By observing how the system responded to an energy perturbation, which here they initiated using patterned light, the team was able to both confirm that the predicted phonons appeared and measure their properties. The speed of sound in the lattice is related to the phonons' presence.

The team found the speed of sound in their rubidium-atom lattice to be $16 \mathrm{~cm} / \mathrm{s}$, about double the speed of a giant tortoise. If it were possible to put your ear to the atoms, you would hear them vibrate at around $1 \mathrm{kHz}$, Lev says. "Of course, you can't do that because the system is in a vacuum chamber and it's really tiny," he jokes. "But we can take pictures of the atoms."

Images taken using the cavity—which also works as a quantum gas microscope-showed that the trapped rubidium atoms formed a vibrating supersolid state, a phase of matter that simultaneously displays crystalline order and superfluid flow.

The demonstration is an "experimental breakthrough," says Victor Galitski, a physicist at the University of Maryland. "It both opens up new avenues for quantum simulation of previously inaccessible phenomena in solid-state materials and potentially may give rise to [researchers uncovering] completely new physics with no analog elsewhere." Elizabeth Goldschmidt, a physicist at the University of Illinois Urbana-Champaign, echoes that opinion. She notes that the ability to turn on phonons in ultracold atoms systems "adds a new feature to the toolkit" for these experiments. "The role of phonons in real crystals is both central to many of their properties and extremely difficult to model in detail. Thus, studying phonons in a much simpler system like this can elucidate new and exciting physics that is highly relevant to many real materials and systems," she says.

Lev agrees. By tweaking the system, he thinks that the approach might be able to create states that emulate the behavior of exotic solid-state superconductors. The system could also create other states of matter that have no direct condensed-matter counterpart, such as a predicted stripy Bose-Einstein condensate that resembles a type of liquid crystal. Lev and his colleagues are also working on using the photon-atom interactions to create spin glasses, or collections of spins that align randomly in a solid. Those structures are promising tools for creating neuromorphic computers, which essentially act like artificial brains by operating in a manner that mimics how the brain sends, receives, and processes signals. "I think that what we have created is this really fertile experimental tool that gives us a brand-new way to shape how quantum particles can interact and organize themselves," Lev says. "It opens up new directions for accessing nonequilibrium quantum matter, a realm that deserves much more exploration."

Katherine Wright is the Deputy Editor of Physics. 